\title{
First Meeting of the WHO THELEP* Scientific Working Group
}

The first meeting of the THELEP Scientific Working Group (SWG) was held in Geneva in April 1977. This meeting had been preceded by 2 earlier THELEP meetings, that of the Planning Committee held in April 1976, and the meeting of the Screening Committee in December 1976. These meetings had established as areas of priority field studies of the prevalence of dapsone resistance, clinical trials in lepromatous leprosy, relevant laboratory investigations and the development of new chemotherapeutic agents, and reviewed applications for support of proposals related to these objectives.

The 78-page report of the first THELEP SWG meeting (WHO document TDR/SWG-THELEP(1)/77.3), which is available on request to the World Health Organization (1211 Geneva 27, Switzerland) includes the "Standard Protocol for Chemotherapeutic Trials in Lepromatous Leprosy". This was prepared by the SGW for use in THELEP-supported studies, and contains a wealth of information on the chemotherapy of leprosy as well as much helpful advice on the treatment of the individual patient.

In the introduction to the report the evidence for the inadequacy of presentday treatment of lepromatous leprosy is reviewed. Present chemotherapeutic methods appear to be inadequate primarily because they fail to prevent the emergence of drug-resistant Mycobacterium leprae and because they appear incapable of eradicating persisting viable bacilli. As a consequence patients may relapse during continued treatment or after the termination of very lengthy periods of chemotherapy. The serious shortcomings in the chemotherapy of lepromatous leprosy appear to have resulted not only from a shortage of effective drugs but also from a lack of knowledge of how to use existing drugs in the most effective way.

One of the major objectives of the THELEP programme is therefore to develop more effective therapeutic regimens for the treatment of lepromatous leprosy, and it was to this end that the Standard Protocol was prepared. Estimates of the efficacy of currently available drugs are largely based on measurements in the footpads of immunologically normal mice of the proportion of viable $M$. leprae recovered from skin biopsy specimens of lepromatous leprosy patients obtained at intervals during therapy with these drugs. These studies have demonstrated that rif ampicin is maximally effective,

\footnotetext{
* "THELEP" is the designation of the Programme for Research in Chemotherapy of Leprosy, which, together with IMMLEP (Programme for Research in Immunology of Leprosy), represents at this time the leprosy component of the WHO Special Programme for Research and Training in Tropical Diseases (TDR).
} 
reducing the proportion of viable organisms below the threshold of detectability within a few days of a single 1200 - or $1500-\mathrm{mg}$ dose, and suggest no drug combination including rifampicin may be expected by this test to appear more effective than rifampicin alone. A few studies using immunosuppressed mice, in which smaller proportions of viable $M$. leprae may be recognized, have demonstrated the survival of drug-susceptible, "persisting" organisms after years of monotherapy with a number of drugs including rifampicin, and after months of therapy with rifampicin and dapsone in full daily dosage. The principal objective of the chemotherapeutic trials conducted according to this standard protocol is to minimize the proportion of persisting $M$. leprae, and, at the same time to prevent the multiplication of drug-resistant organisms. Furthermore it is believed that those regimens which minimize the number of persisting leprosy bacilli will also be the most eff ective in preventing the subsequent emergence of drug-resistant organisms. No trial of drug combinations, especially those including rifampicin, will yield meaningful results unless persisting $M$. leprae are measured. Consequently, the inoculation of immunosuppressed rodents is mandatory for compliance with the THELEP standard protocol.

THELEP intends eventually to mount chemotherapeutic trials among three categories of lepromatous leprosy patients: untreated patients with fully drugsensitive $M$. leprae; patients with proven dapsone-resistant leprosy bacilli; patients who have already responded to a period of monotherapy with dapsone and who may therefore be presumed to harbour larger proportions of dapsoneresistant organisms than are normally encountered in previously untreated patients.

The Standard Protocol prepared by the THELEP SWG has been designed to assess the relative efficacy of different regimens in treating these 3 types of patients, and endeavours to elicit the maximum amount of information while exposing patients to the minimum of risk. The Protocol envisages the trials as having 2 phases - an initial short-term phase of 3 months' intensive treatment followed by a long-term phase of less intensive treatment of 21 months' duration. Depending upon the results of these first 2 phases, a third phase may be envisaged. If no persisting $M$. leprae are detected after treatment for 24 months with one or more regimens, therapy could safely be withdrawn from half of the patients, and all of the patients observed thereafter for a minimum of 5 years. In those patients in whom persisters are detected after 24 months, the effect of a change of regimen on the persisters could be measured.

Eight potential regimens were selected for evaluation in previously untreated patients:

Regimen $\mathrm{A}_{1}$ : Dapsone, $100 \mathrm{mg}$ daily for the duration of the trial; Rifampicin, $600 \mathrm{mg}$ daily for the duration of the trial; Clofazimine, 50 or $100 \mathrm{mg}$ daily for the duration of the trial.

Regimen $\mathrm{A}_{2}$ : This identical to Regimen $\mathrm{A}_{1}$, except that prothionamide, $500 \mathrm{mg}$ daily, is substituted for clof azimine.

Regimen B: Dapsone, $100 \mathrm{mg}$ daily for the duration of the trial; Rifampicin, $600 \mathrm{mg}$ daily for the duration of the trial.

Regimen C: Dapsone, $100 \mathrm{mg}$ daily for the duration of the trial; Rifampicin in a single initial $1500 \mathrm{mg}$ dose. 
Regimen $\mathrm{D}_{1}$ : Dapsone, $100 \mathrm{mg}$ daily for the duration of the trial; Rifampicin in a single initial $1500 \mathrm{mg}$ dose; Clofazimine, 50 or $100 \mathrm{mg}$ daily for the first 3 months.

Regimen $\mathrm{D}_{2}$ : This is identical to Regimen $\mathrm{D}_{1}$ except that prothionamide, $500 \mathrm{mg}$ daily, is substituted for clofazimine.

Regimen $\mathrm{E}_{1}$ : Dapsone, $100 \mathrm{mg}$ daily for the duration of the trial; Rifampicin, $900 \mathrm{mg}$ once weekly for the first 3 months; Clofazimine, 50 or $100 \mathrm{mg}$ daily for the first 3 months.

Regimen $\mathrm{E}_{2}$ : This is identical to Regimen $\mathrm{E}_{1}$, except that prothionamide, $500 \mathrm{mg}$ daily, is subsituted for clofazimine.

Four regimens were chosen for potential studies in patients with dapsoneresistant leprosy utilizing daily treatment with clofazimine (50 or $100 \mathrm{mg}$ ) or thiacetazone $(150 \mathrm{mg})$ supplemented by the addition of rifampicin or/rifampicin plus prothionamide. The same 4 regimens supplemented with $100 \mathrm{mg}$ dapsone daily were selected for trials involving patients who had already responded to an initial period of treatment with dapsone alone.

All THELEP-sponsored trials require the approval of governmental and institutional authorities. Approximately 30 patients will be recruited into each of the regimens being studied in the collaborating centres. Patients who agree to participate in the trials should be at least 15-years-old and in apparently good health. They should have LL (LLp) or LI (LLs) leprosy by clinical and histopathological classification and have Mitsuda reactions of less than $3 \mathrm{~mm}$ in diameter. Patients will however be excluded if they have tuberculosis requiring treatment, a life-shortening disease such as cancer, severe diabetes, severe hypertension, renal, hepatic or cardiac diseases or other significant psychological or organic disease that might adversely affect their compliance with therapy or follow-up. Pregnant patients will also be excluded if they have a history of ENL.

The clinical investigator will carry out a full clinical examination of each patient on his entry into the trial, which will include a complete clinical history and physical examination, anatomical silhouettes that map and give measurements of skin lesions, nerve enlargements, and sensory and joint changes. Pretreatment biopsies will be taken to provide $M$. leprae for inoculating immunologically normal mice in order to establish the presence of viable leprosy bacilli and to determine their susceptibility to dapsone. Further biopsies will then be taken after 3, 12 and 24 months treatment to provide bacilli to inoculate both immunologically normal mice and immunologically suppressed rodents. The results obtained from the biopsies taken at 3 months should demonstrate that the patient's leprosy bacilli have been killed at a satisfactory rate during the initial phase of intensive treatment and confirm that when these combinations of drugs are given they do not antagonize each other's bactericidal action.

The most crucial evidence for assessing the relative potency of the regimens will however be derived from the infectivity for immunologically suppressed rodents of inocula containing large numbers of leprosy bacilli prepared from biopsies obtained after 3, 12 and 24 months treatment, because these determinations should enable measurement and comparison of the proportions of $M$. leprae that survive the initial phase of intensive treatment and persist 
during 9 to 21 months of continuing treatment. Portions of the biopsies will also be fixed and processed for histopathological classification according to the Ridley and Jopling system and for measurement of the logarithmic biopsy index.

The therapeutic progress of the patients will also be monitored by carrying out regular clinical examinations, and by measuring the bacterial indices in smears taken from 6 skin sites and prepared from nasal secretions. Throughout the course of treatment the occurrence of ENL and other leprosy reactions will be carefully noted and described, and patients will be regularly questioned to ensure that symptoms suggesting adverse reactions to the drugs they are being prescribed are not missed. Samples of blood, urine and stool will also be collected at specified intervals for routine laboratory studies to monitor haematological parameters and hepatic and renal function, and assess the regularity with which the dapsone prescribed has been ingested.

The THELEP SWG's report also includes with the Standard Protocol detailed appendices describing how the clinical examinations should be conducted, the classification of LL and LI leprosy and the lepromin test procedure. Other appendices describe the methods to be employed for preparing the skin and nasal smears and the measurement of their bacterial indices. Detailed descriptions are also given of the techniques to be used for obtaining the skin biopsies, and of how they should be prepared, packaged and shipped to the reference laboratories for animal inoculation and histopathological examination. The methods for inoculating immunologically normal and immunologically suppressed mice are also described in detail, together with the procedures to be used for the subsequent enumeration of $\boldsymbol{M}$. leprae in their footpads. The urine-test method to be used for monitoring the ingestion of dapsone is described in full. Further appendices provide guidance on the management of ENL and reversal reactions, the monitoring of adverse reactions to drugs and the criteria to be used for removing patients from the controlled clinical trials because of the possibility of drug toxicity.

The report also includes brief descriptions of the facilities and patients available for clinical trials at treatment centres in 10 countries. Summaries are also included of the deliberations of the 2 informal subgroups that were formed during the THELEP SWG meeting. One subgroup considered methods for measuring the prevalence of dapsone resistance and the conduct of clinical trials of non-lepromatous leprosy, while the other subgroup designated 5 areas of laboratory-based research activity that could aid the development and screening of new antileprosy drugs or the more effective use of combinations of established drugs.

\section{Participants of the THELEP Scientific Working Group at its First Meeting, April 1977}

Dr J. BARZELATTO, Special Programme for Research and Training in Tropical Diseases, WHO, Geneva, Switzerland.

Dr LE KINH DUE, Clinique de Dermatologie et de Vénérologie, Hôpital Bach Mai, Hanoi, SRVN.

Dr G. A. ELLARD, MRC Unit for Laboratory Studies of Tuberculosis, Royal Postgraduate Medical School, London W12 0HS, England (Rapporteur). 
Dr V. A. EVSTRATOVA, Leprosy Research Institute, Astrakhan, U.S.S.R.

Dr A. H. FIELDSTEEL, Stanford Research Institute, Menlo Park, California 94025, U.S.A.

Dr R. GELBER, U.S. Public Health Services Hospital, 15th Avenue and Lake Street, San Francisco, California 94118 , U.S.A.

Dr L. M. HOGERZEIL, Victoria Hospital, Dichpalli, Nizambad District, Andhra Pradesh, India.

Dr T. ITO, Research Institute for Microbial Diseases, Osaka University, Yamada-kami, Suita Osaka, Japan.

Dr C. G. S. IYER, Central Leprosy Teaching and Research Institute, Chingleput, Tamil Nadu 603001 , India.

Dr KYAW LWIN, Ministry of Health, 34/42 Strand Road, Rangoon, Burma.

Dr J. LANGUILLON, Institut de Léprologie Appliquéé, B.P. 11.023 CD Annexe, Dakar, Senegal.

Dr D. L. LEIKER, Royal Tropical Institute, Department of Tropical Hygiene, Mauritskade 63, Amsterdam, Netherlands.

Dr L. LEVY, Department of Comparative Medicine, Hebrew University-Hadassah Medical School, Jerusalem, Israel.

Dr D. V. OPROMOLLA, Hospital Lauro Sousa Lima, 17100 Bauru, Sao Paulo, Brazil.

Dr S. R. PATTYN, Universitaire Instellung Antwerpen, 2610 Wilrijk, Belgium.

Dr J. M. H. PEARSON, MRC Leprosy Project, P.B. 1005, Addis Ababa, Ethiopia.

Dr R. J. W. REES, National Institute for Medical Research, Mill Hill, London NW7 1AA, England (Chairman).

Dr E. J. SAERENS, 65 Av. de la Nivéole, $B-1020$ Brussels, Belgium.

Dr J. K. SEYDEL, Forschunginstitut Borstel, 2061 Borstel, Federal Republic of Germany.

Dr C. C. SHEPARD, Leprosy and Rickettsia Branch, Center for Disease Control, Atlanta, Georgia 30333, U.S.A.

Dr L. VILLALBA-PIMENTEL, Instituto Nacional de Dermatologia, Apartado 4043, Caracas 101 , Venezuela.

Dr M. F. R. WATERS, National Institute for Medical Research, Mill Hill, London NW7 1AA, England.

Dr J. S. YAW ALKAR, Ciba-Geigy Limited, 4002 Basle, Switzerland.

\section{SECRETARIAT}

Dr H. SANSARRICQ, Leprosy Unit, WHO, Geneva, Switzerland.

Dr J. WALTER, Leprosy Unit, WHO, Geneva, Switzerland.

Dr A. O. LUCAS, Director, Special Programme for Research and Training in Tropical Diseases, WHO, Geneva, Switzerland.

Dr E. DE MAAR, Special Programme for Research and Training in Tropical Diseases, WHO, Geneva, Switzerland.

Dr K. L. HITZE, Tuberculosis and Respiratory Infections Unit, WHO Geneva, Switzerland.

Dr L. REY, Schistosomiasis Unit, WHO, Geneva, Switzerland.

Mr T. K. SUNDARESAN, Health Statistical Methodology Unit, WHO, Geneva, Switzerland. 